\title{
Special issue: best papers of APWeb 2010
}

\author{
Wook-Shin Han • Divesh Srivastava • Ge Yu
}

Received: 12 July 2011

Accepted: 19 July 2011 /Published online: 17 August 2011

(C) Springer Science+Business Media, LLC 2011

This issue of World Wide Web includes revised and expanded versions of two papers from the 12th Asia-Pacific Web Conference (APWeb), which took place in Busan, Korea on 6-8 April, 2010.

The papers were selected as best papers based on their overall technical quality. They were selected by the program committee co-chairs, Wook-Shin Han, Divesh Srivastava, and Ge Yu.

The first paper, "A Space and Time Efficient Algorithm for SimRank Computation," by Weiren Yu, Wenjie Zhang, Qing Zhang, Xuemin Lin, and Jiajin Le proposes novel optimization techniques to accelerate SimRank Computation. In addition, the authors develop a reordering scheme to achieve both faster convergence rate and I/O efficiency. They also perform extensive experiments using many data sets to demonstrate the efficiency of the proposed techniques.

The second paper, "Quick Identification of Near-Duplicate Video Sequences with Cut Signature," by Qing Xie, Zi Huang, Heng Tao Shen, Xiaofang Zhou, and Chaoyi Pang is about fast identification of duplicate video sequences. The authors consider the issue of how to efficiently and effectively detect near-duplicate video sequences over continuous video streams, and propose a novel compact signature called CutSig to approximate the video structure. They exploit CutSig to efficiently filter candidates. They empirically validate the effectiveness and efficiency of the proposed method using real video streams.

W.-S. Han $(\bowtie)$

Kyungpook National University, Daegu, South Korea

e-mail: wshan@knu.ac.kr

D. Srivastava

AT\&T Labs-Research, 180 Park Avenue, Bldg 103, Florham Park, NJ 07932, USA

e-mail: divesh@research.att.com

G. $\mathrm{Yu}$

School of Information Science and Engineering, Northeastern University, Wenhua Road 3-11, Heping District, Shenyang 110004, China

e-mail: yuge@ise.neu.edu.cn 